\title{
Magnetically Driven Middle Ear Ossicles for Optical Measurement of Vibrations in an Ear with Opened Eardrum
}

\author{
John Peacock ${ }^{\mathrm{a}}$, Magnus von Unge ${ }^{\mathrm{b}}$ and Joris Dirckx ${ }^{\mathrm{a}}$ \\ ${ }^{a}$ Laboratory of Biomedical Physics, University of Antwerp \\ Groenenborgerlaan 171, B-2020, Antwerp, Belgium \\ ${ }^{b}$ Department of Otorhinolaryngology, Akershus University Hospital, University of Oslo \\ Oslo, Norway
}

\begin{abstract}
Vibrations of the middle ear ossicles are easily measured by means of laser vibrometry. However, exposing the ossicles requires the removal of the eardrum, with the result that the ossicles can no longer be stimulated acoustically. To overcome this we devised a new set up in which the ossicles can be driven magnetically. After measuring the response of the eardrum to an acoustic signal, we then remove the eardrum and attach a small magnet to the exposed manubrium (the part of the first auditory ossicle, the malleus, which is normally attached to the eardrum). An electromagnetic excitation coil is then used to drive the magnet, and the output to the coil adjusted until the vibration of the manubrium, as measured by the vibrometer, matches that measured in response to the acoustic signal. Such a set-up has uses in research on middle ear mechanics, such as the measurement of non-linearities in their response, as well as applications in the diagnosis of middle ear conditions such as the fixation of the ossicles by otosclerosis, or in chronic otitis media. We describe our set up in which the vibrometer unit is attached to a surgical microscope, offering accurate positioning of the laser beam. We discuss the viability of our method and its future potential by presenting some measurements on artificially fixated ears.
\end{abstract}

Keywords: laser vibrometry, ear surgery

PACS: $42.62 . \mathrm{Be}$, 87.64.G, 87.85.M

\section{INTRODUCTION}

Chronic inflammation of the middle ear (Chronic otitis media) is a fairly common disease and a major cause of hearing impairment worldwide [1]. Amongst the structural changes caused by the condition is a reduction in the mobility of the middle ear ossicles. This reduction is an important factor in helping an ear surgeon determine the best course of treatment. However, a means of accurately measuring ossicular motion is lacking. The only method available today is to manually press on the ossicles and observe their motion. This can show that the ossicles are completely fixed, but in cases of partial fixation it offers little help. A method of accurately measuring the ossicles could, therefore, be of much use.

Laser vibrometry is one technique which would permit accurate measurement. However, gaining a clear view of the ossicles presents some challenges. If we are measuring on a preserved temporal bone in, for instance, studies of middle ear mechanics, access can be provided by drilling a hole at the back of the temporal bone. This method is, obviously, not possible for measurements on living patients, where the only way to access the ossicles is through the ear canal. This poses a problem since, in order to stimulate the ossicles, one requires an intact eardrum; without the eardrum the ossicles won't respond to acoustic stimulation. But in order to expose the ossicles through the ear canal, one would first need to remove the eardrum. After the eardrum is removed, any measurements we do make would be meaningless.

A method is required that gives us access to the ossicles for measurement, yet allows us to stimulate them and have them respond in the same way they would to an acoustic signal. This paper describes a possible solution.

After removal of the eardrum, a small magnet is attached to the manubrium (the part of the first auditory ossicle, which is normally attached to the eardrum) and, by means of an electromagnetic excitation coil, the magnet is made to vibrate and so induce motion in the ossicles. We describe our method and set-up, in which the vibrometer unit is attached to a surgical microscope. We discuss the viability of the method and its future potential in middle ear surgery. 


\section{SET-UP AND METHOD}

\section{Coupling the Vibrometer to the Microscope}

Fast and accurate positioning of the laser beam was achieved by the attachment of the vibrometer to a surgical microscope. Both preparation and measurement are thus achieved with the same apparatus, and we can see exactly what the laser beam will be able to reach when preparing the object to be measured. A drawing of this set up is shown in figure 1.

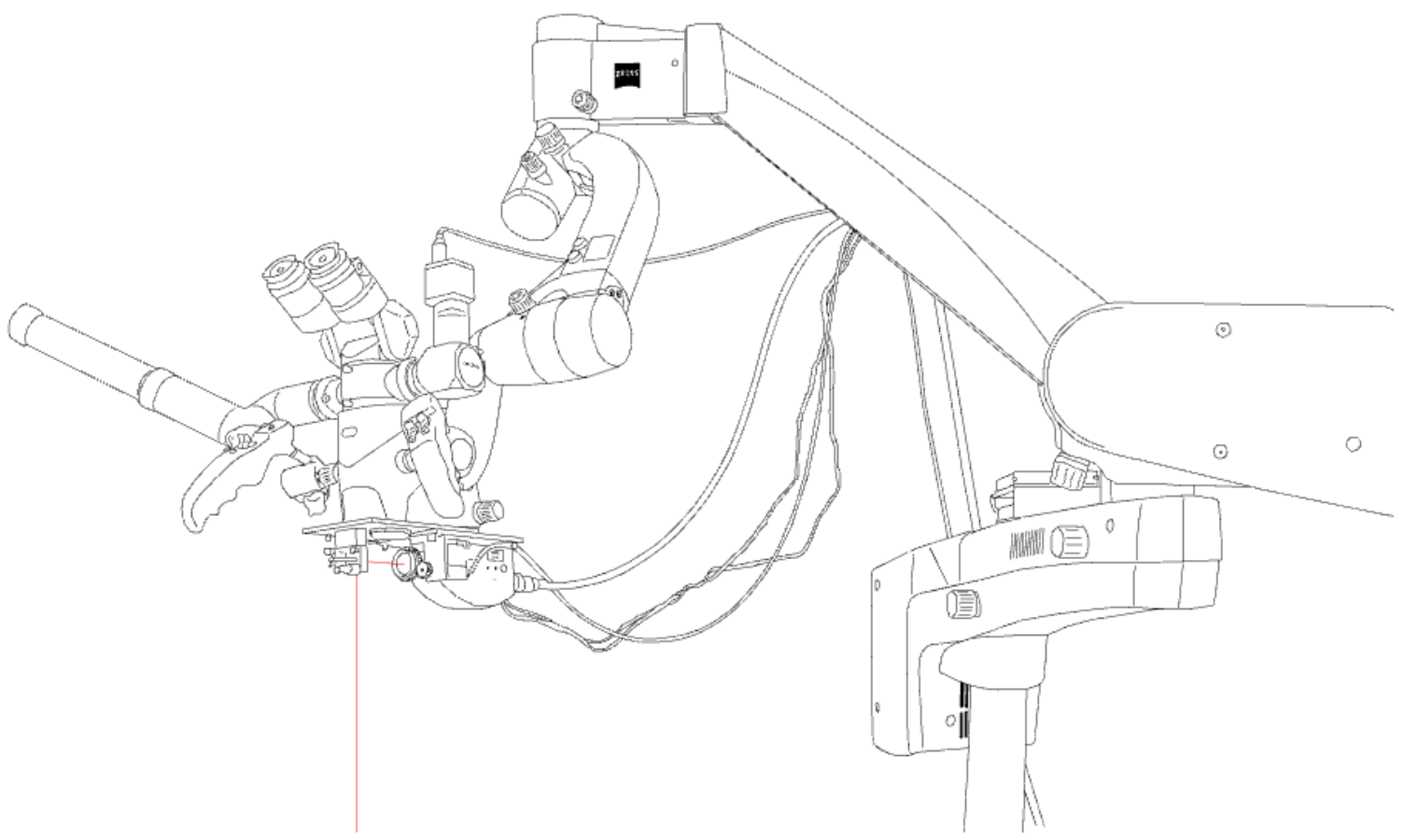

FIGURE 1. Drawing of the microscope with the vibrometer attached. The red line depicts the path of the laser beam.

It might be expected that the long arm of the microscope would introduce additional vibrations that might interfere with measurements, or, at the very least, increase the noise levels. This did not turn out to be the case, at least not in the frequency range which is physiologically relevant. If one repositions the microscope or moves it in any way then the effects are clearly visible in the vibrometer data. But whilst it is kept still we see no adverse effects. If the microscope does have to be repositioned, it is only necessary to allow it a few seconds to 'settle' before resuming measurements, a delay which causes little disruption.

In our set-up we reduced the need to touch or manipulate the microscope further by allowing fine positioning and focusing of the laser beam to be achieved without touching the apparatus (see figure 2). Piezo motors are used to change the position of a small mirror, and thus move the laser beam to different positions. Another motor is set such that it will adjust the focusing of the laser. The operation of these motors is done by a joystick, allowing the operator to make adjustments without taking their eyes from the microscope.

This set up allows to quickly and accurately move the laser beam from one ossicle to another, a procedure that might otherwise have rendered the proposed clinical application unworkable. 


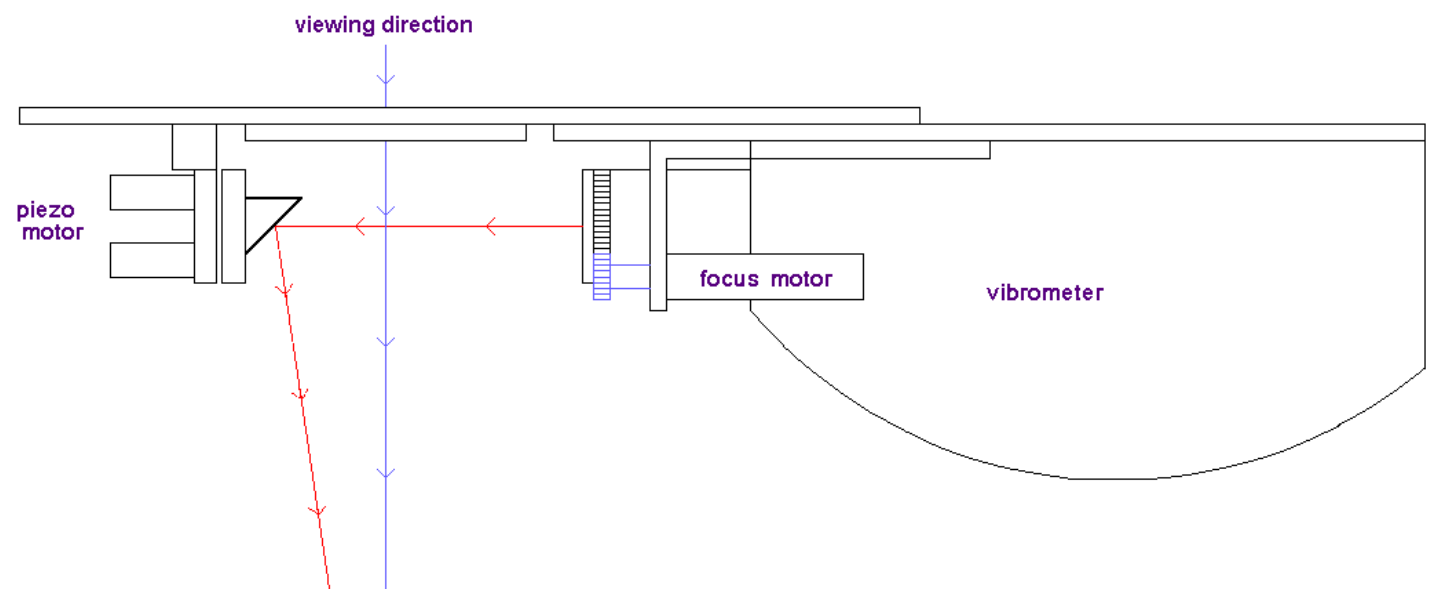

FIGURE 2. Detail of the vibrometer unit attached to the microscope. Focussing and fine positioning of the laser beam can be done without touching the apparatus and so disturbing the system. The red line depicts the path of the laser beam, whilst the blue line depicts the line of sight of the microscope operator.

\section{Magnetic Stimulation of the Ossicles}

Before removing the eardrum a measurement was made on it. It was stimulated with an acoustic signal and its vibrations recorded by the vibrometer at the umbo; the point at which the tip of the manubrium is attached to the eardrum. Next, after this measurement was complete, we removed the eardrum and attached a small magnet to the manubrium. This was then stimulated by means of a pancake coil with the magnet at its centre. We then measured at the tip of the manubrium, the same position as was measured with acoustic stimulation, and the vibration response we got was compared with that which we achieved with acoustic signal. By comparing the two, we adjusted the output to our coil until they matched.

Once we see that they match, and thus can be sure that the tip of the manubrium was behaving the same way as with the eardrum intact, we can be sure that the rest of the ossicles are likewise behaving the same as before.

The output to the coil can be adjusted to keep the ossicular response to magnetic and acoustic stimulation within $1 \mathrm{~dB} \mathrm{svl}^{1}$. Figure 3 shows measurements made on a preserved (frozen) human temporal bone. The behaviour of frozen bones is considered to be a good match to fresh ones [2].

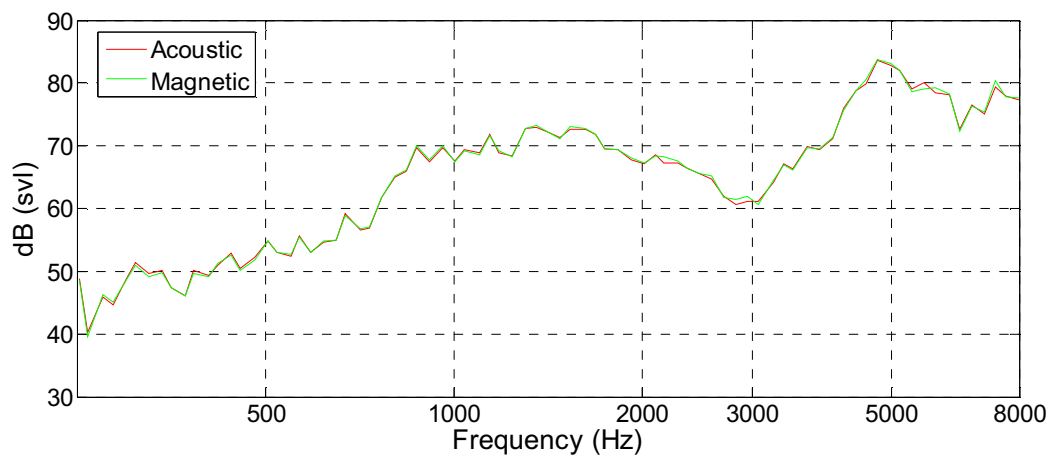

FIGURE 3A. The calibration of the coil. The output of the coil is easily adjusted to keep the vibration response within $1 \mathrm{~dB}$ of that obtained acoustically.

${ }^{1} \mathrm{SVL}$, or sound velocity level (also known as particle velocity level), is calculated with a reference velocity of $5 \mathrm{x} 10-8 \mathrm{~m} / \mathrm{s}$. i.e. $20 \log 10(\mathrm{v} / 5 \mathrm{x}$ $10-8)$, where $\mathrm{v}$ is the measured velocity. 


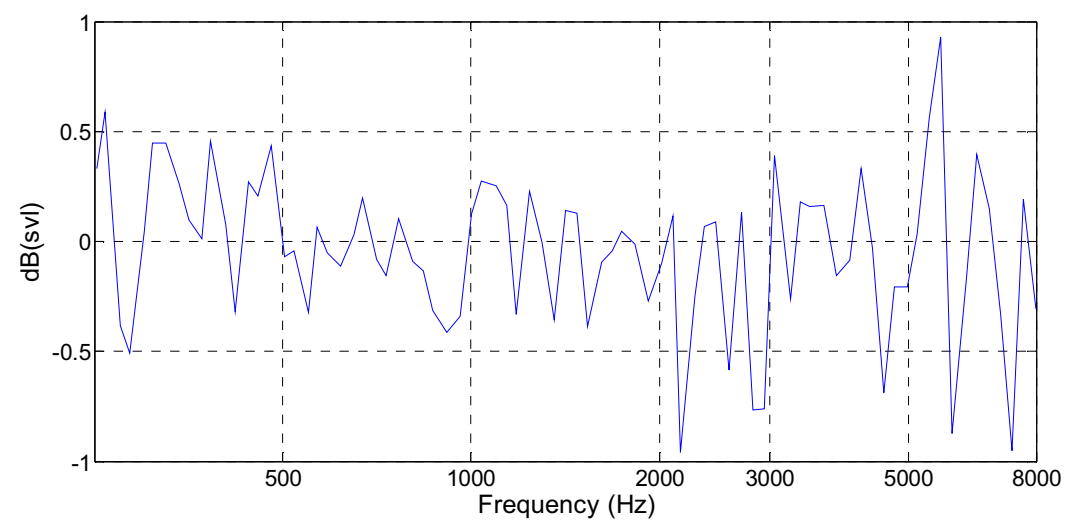

FIGURE 3B. The difference between the acoustically and magnetically elicited vibration response.

Keeping the response of the ossicles within $1 \mathrm{~dB}$ is easily achievable, and is well within the variation required for extracting useable data. Some variation, up to a few $\mathrm{dB}$, can be expected over repeated measurements of the same point due to physiologic variability, but this variation would still provide us with useable data.

To check the reproducibility of the measurements over time, we repeated measurements 10 times over a ten minute period using a vinyl membrane as a test object. An artificial membrane was chosen to exclude any changes due to the object, and purely test the set up. As such a membrane has much sharper resonance peaks, as compared to an eardrum, results are even more perceptible to apparatus changes than in our proposed application. Figure 4 shows the vibration response and the maximal spread of the measured values over time.

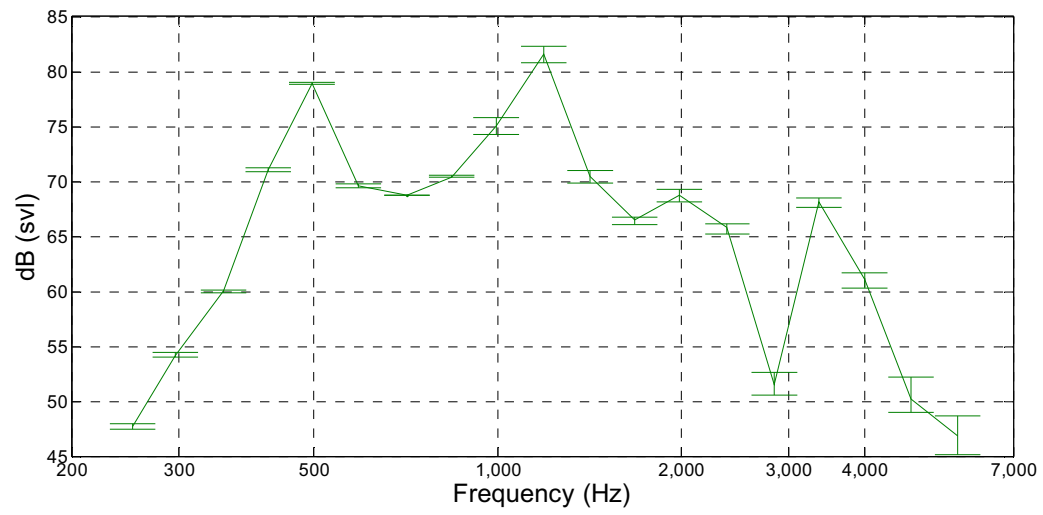

FIGURE 4. Response of a vinyl membrane with the magnet attached to its surface. The graph shows repeated measurements taken over a period of ten minutes: with the solid line corresponding to the average values, and the error bars showing the largest and smallest value at each frequency.

The largest variations occur at the higher frequencies. But even so, the largest variation for a single frequency is only $3 \mathrm{~dB}$, still within the limits we require. Measurements over a period of 10 minutes are shown here to illustrate the stability in the method. Although it is true that any clinical measurements would require a much shorter time, this does not imply that they would be more accurate. The range of values seen here is not due to changes over time; measurements over a shorter or longer period would give the same spread of values. So long as the apparatus is not moved, and the measured object remains stable, the measurements are very repeatable. 


\section{The Effects of Changes in the Position of the Coil}

One other factor which may affect the use of this technique lies in how changes in the coil's position, with respect to the magnet, affect the output. This is particularly relevant for any potential clinical application, where one cannot expect a living patient to remain absolutely still for the duration of the procedure. Two factors reduce the possibility of movement: the first, that any patient will be anaesthetised and second, that the time from calibration to completing a measurement is very short (it could take much less than a minute to calibrate and measure). So it may be expected that, in practice, any change in the position of the coil will be limited to a few mm in difference and a few degrees in tilt. In order to accurately determine the effects of any changes, we performed measurements on a vinyl membrane, shown in figures 5 and 6.

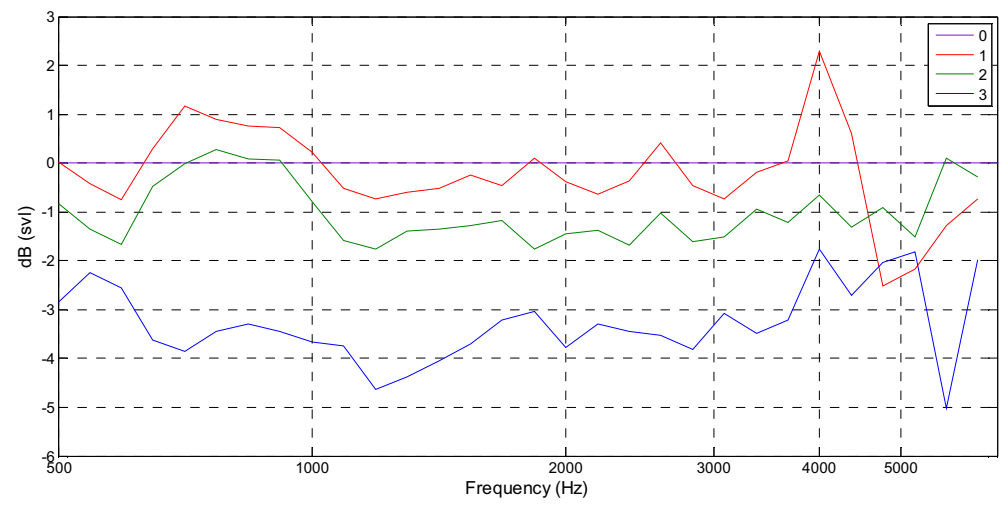

FIGURE 5. Change in the measured response of the membrane after changing the position of the coil. 0 (purple) is the original position when the signal was calibrated. The coil was moved by 1 (red), 2 (green) and 3 (blue) $\mathrm{cm}$.

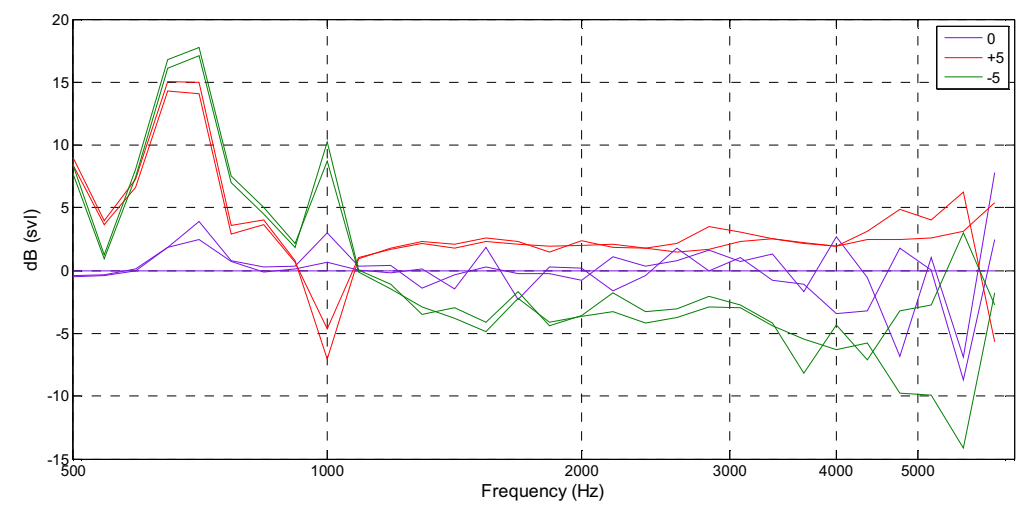

FIGURE 6. Change in the measured response of the membrane after changing the angle of the coil. Two measurements are shown at +5 (red) and -5 (green) degrees from the original position and 3 measurements (purple) are show at the original position.

At most the coil would be expected to move $1 \mathrm{~cm}$ or less from its original position, or to change its orientation by 5 degrees or less. For figure 5 the coil was moved 1, 2 then $3 \mathrm{~cm}$ from its original position, and in figure six it was rotated \pm 5 degrees from its original position.

Changes in the position of the coil show very little change in the response of the membrane. Even at $3 \mathrm{~cm}$ the response is still within $5 \mathrm{~dB}$ of the original signal. At the more likely change of $1 \mathrm{~cm}$, the response remains within $1 \mathrm{~dB}$ of the original signal, except at higher frequencies where is reaches around $2 \mathrm{~dB}$, which is still below the $3 \mathrm{~dB}$ 
variations seen in figure 4 . We can reasonably conclude that changes in the coil's position, of around $1 \mathrm{~cm}$, have negligible effect on the repeatability of the measurements.

Figure 6 tells a slightly different story, with changes in the orientation of the coil proving much more significant. For the most part, the measured response remains within $5 \mathrm{~dB}$ of the original signal. Only at the low or high frequencies does it rise above. Much of this change can be attributed to the changed orientation of the coil, however the process of repositioning the apparatus likely introduced some other differences.

The rather large changes at 500 and $1000 \mathrm{~Hz}$ are due to the sharp resonances of the vinyl membrane. An eardrum, on the other hand, does not show such sharp peaks. At high frequencies the technique does become more perceptible to angular changes of the coil. In the frequency range up to $4 \mathrm{kHz}$, angular changes in the coil position up to $5^{\circ}$ result in a response change of less than $5 \mathrm{~dB}$. In the practical application, the coil angle can be well maintained within a range of a few degrees.

\section{Measurements on Artificially Fixated Ears}

Figure 7 shows the results of measurements on a human temporal bone. In order to simulate different degrees of ossicle fixation, glass ionomer luting cement was used. Three stages of fixation were measured with fix 1 being the least fixed and fix 3 being the most fixed. Measurements were made at three points: the umbo (tip of the manubrium), the incus, and the stapes. The stapes measurements differed little from the incus, and so are not shown here. One further measurement was made on the temporal bone in order to determine the background noise level, and to make certain that the ossicles were being induced to move independently of the rest of the apparatus.

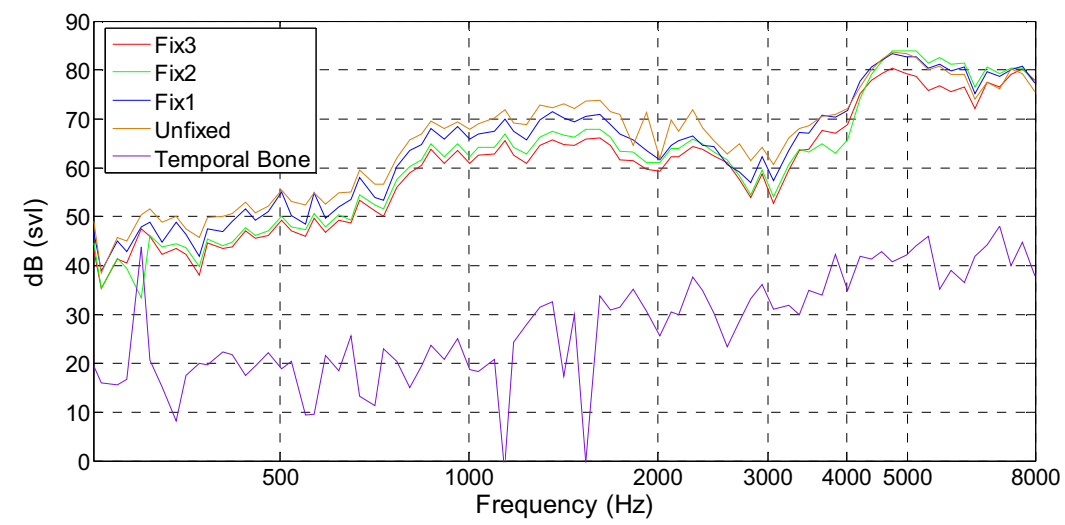

FIGURE 7A. The response of the umbo after various degrees of fixation. The measurement on the temporal bone shows the noise floor.

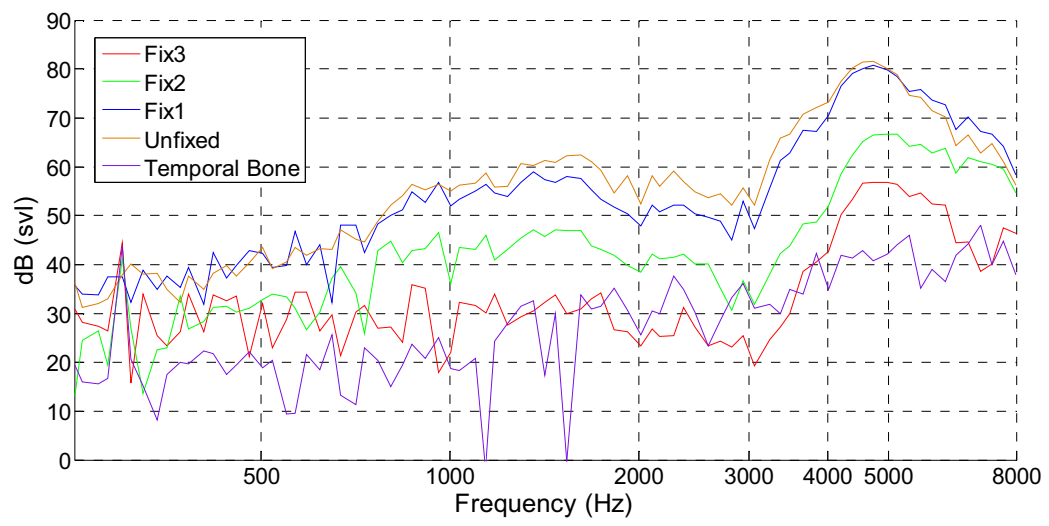

FIGURE 7B. The response of the incus after various degrees of fixation 
From figure 7A we see that umbo velocity is reduced very little as a result of the ossicle fixation, suggesting that vibrometry measurements on the eardrum alone have little clinical relevance. Figure $7 \mathrm{~b}$, showing the results obtained on the incus, is, however, different. The ossicles are not equally affected by fixation, with the changes in the incus being much larger than those of the umbo.

Around 4000 to $5000 \mathrm{~Hz}$, the measurements get increasingly noisier with increasing frequency. Likewise, at lower frequencies, they begin to get noisier between 500 and $1000 \mathrm{~Hz}$. However, between these values the data is very clear. The difference in the response of the incus and that of the umbo increases with progressing ossicular fixation, suggesting that information on the size of this gap could be of some clinical use.

\section{SUMMARY AND CONCLUSIONS}

The measurements presented here demonstrate the viability of our method. With the use of magnets, in combination with laser vibrometry, it is possible to stimulate the ear in the same way as with an acoustic signal. Even with small variations in the position of the coil, with respect to the object being measured, the ability to induce accurate and useful measurements on middle ear ossicles remains possible. The measurements presented here indicate that the size of the gap between the vibration response of the incus and that of the umbo gives us some indication of the state of fixation of the ossicles. But further measurements on fresh human temporal bones will be necessary to fully determine the clinical value.

\section{REFERENCES}

1. Prevention of Hearing Impairment from Chronic Otitis Media, WHO/ CIBA Report, 1998, www,who.int/entity/pbd/deafness/en/chronic_otitis_media.pdf

2. Rosowski, J.J., Davis, P.J., Merchant, S.N., Donahue, K.M., Coltrera, M.D., 1990. Cadaver middle ears as models for living ears: Comparisons of middle-ear input immittance. Ann. Otol. Rhinol. Laryngol. 99, 403-412. 
Copyright of AIP Conference Proceedings is the property of American Institute of Physics and its content may not be copied or emailed to multiple sites or posted to a listserv without the copyright holder's express written permission. However, users may print, download, or email articles for individual use. 